\title{
Failure Analysis of CIGS Solar Cells by SEM/EDS
}

\author{
John Konopka ${ }^{1}$ \\ 1. Thermo Fisher Scientific, Madison, USA.
}

Copper, Indium, Gallium, Selenium (CIGS) solar cells are well suited to analysis by SEM/EDS. They consist almost entirely of inorganic materials, and the dimensions of the sample are amenable to SEM/EDS analysis consisting principally of layers 0.4 to 2 microns thick. SEM/EDS analysis of these samples can verify that the compositions and dimensions of the manufactured part are as expected, it can identify variances from specifications, and it can characterize artifacts discovered during observation.

Fracturing, sectioning with a diamond saw and ion milling were tested as methods of sample preparation. Samples were coated with C or Ir to make them conductive for observation in the SEM.

The SEMs used were a Hitachi SU-6600 FESEM and a Thermo Fisher Scientific Q250 both with a Thermo Scientific UltraDry silicon drift detector and NSS EDS analyzer. Ion milling was performed with a Hitachi Ion Mill.

Figure 1 is an image of a fracture surface with selected net counts elemental maps of this view. Figure 2 is an image and selected net counts elemental maps of a cross section of the sample which has been ion milled. Both views clearly show the different layers which constitute the CIGS cell. Both views show that the $\mathrm{S}$ distribution is wrong and that the Ge layer is not well formed in these samples. The ion milled sample provides a much clearer view of the sample structure including the grain structure of the CIGS absorber. Figure 3 is a BSE image of the ion milled sample showing this grain structure.

Simple fracturing of the sample adequately provides a useful cross section for observation. It allowed for measuring the thicknesses of the layers and confirming their uniformity. The resultant rough surface did obscure some details of the sample. It was not possible to select the region of the fracture with any precision because of the toughness of the protective plastic coating on the cell.

Ion milling after fracturing provides a much superior surface for observation. In many cases individual crystal grains are discernible. Ion milling made it possible to detect thin features masked in the fracture face. The disadvantages are the cost of the instrument, the time needed to polish one sample and the limited region available for observation (less than $1 \mathrm{~mm}$ ).

Sectioning by the diamond saw proved unsuccessful. It did allow for precise positioning of the cut surface which was very helpful, but it caused severe fracturing and disruption of the active layers and delamination of these layers from the substrate. 


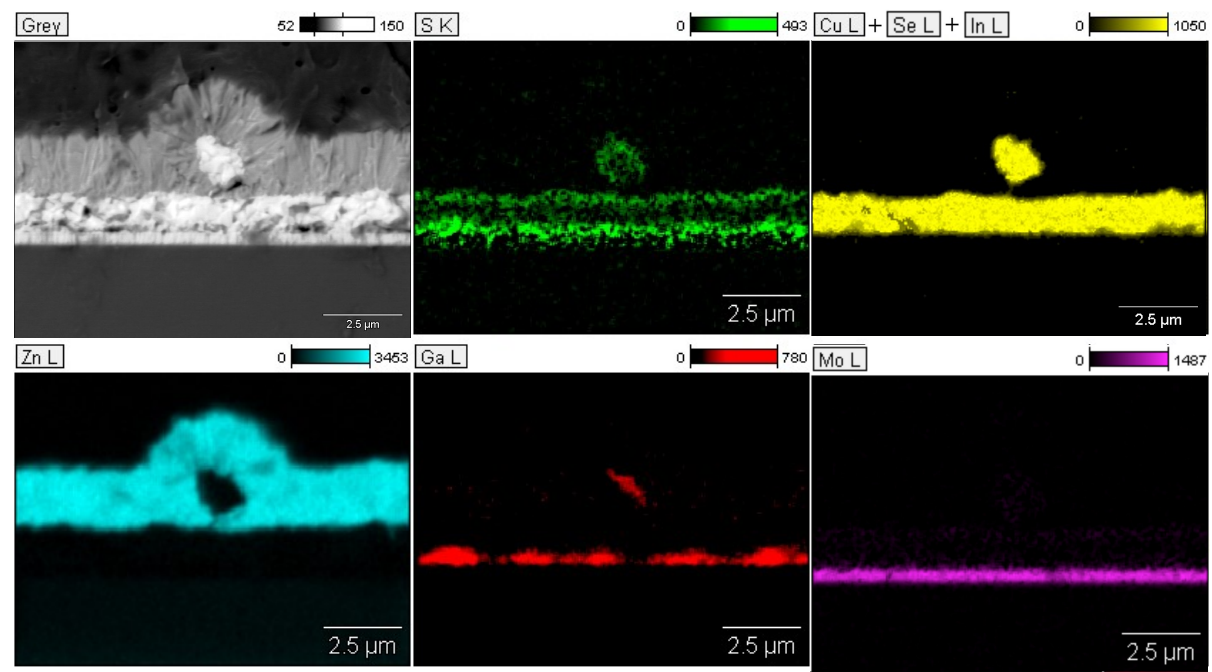

Figure 1. Selected net counts elemental maps from fractured sample of CIGS solar cell. 5kV

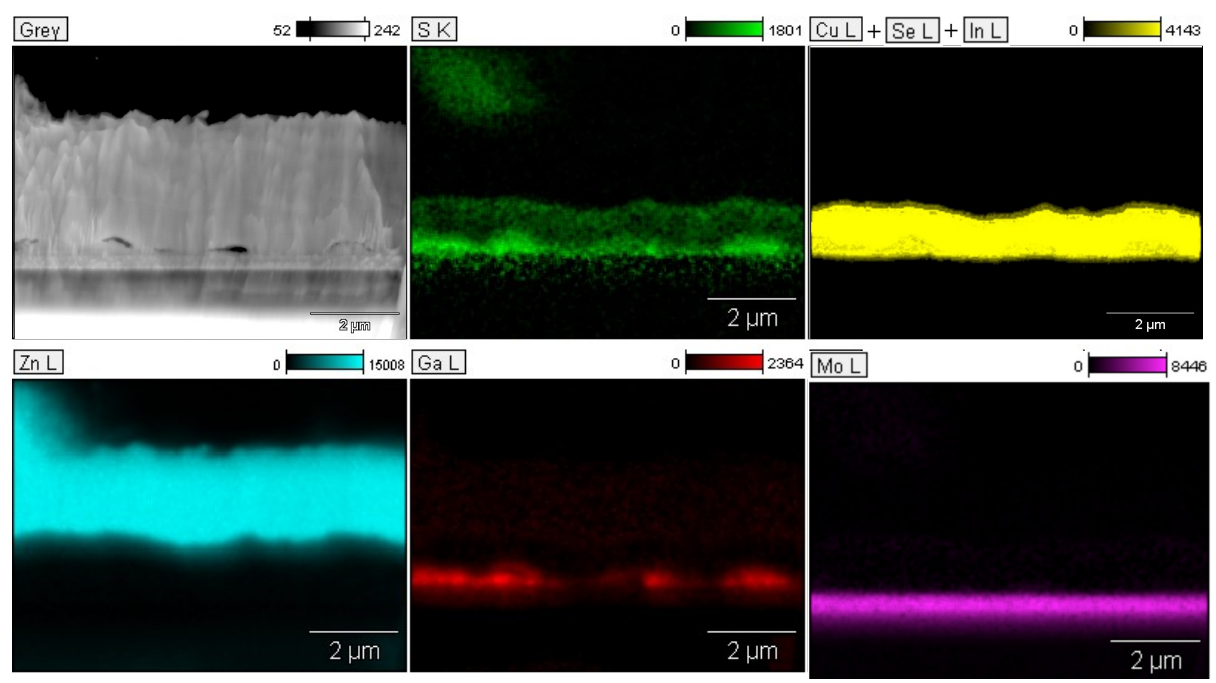

Figure 2. Selected net counts elemental maps from ion milled sample of CIGS solar cell. $7 \mathrm{kV}$

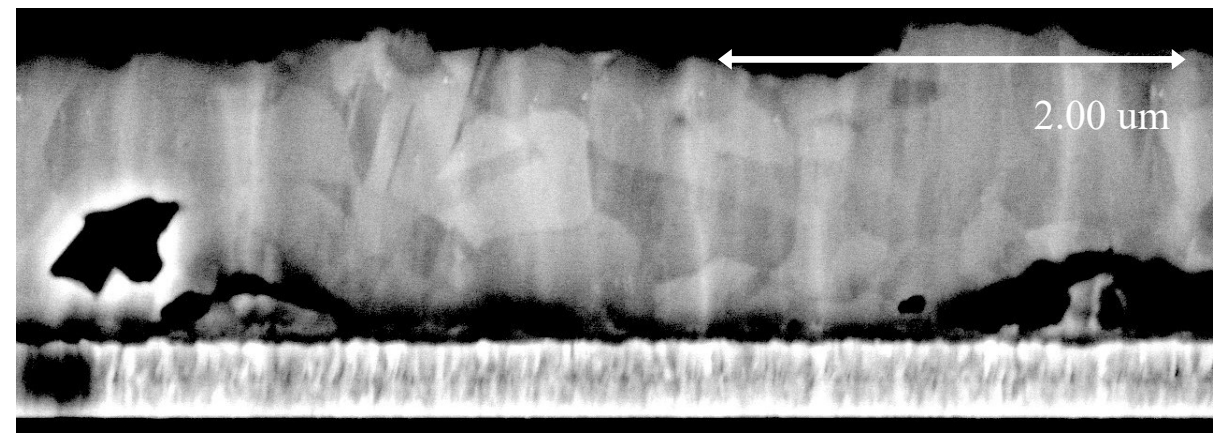

Figure 3. Section of BSE micrograph of ion milled CIGS sample revealing grain structure in central structure. $7 \mathrm{kV}$ 